José Manuel Macário Rebêlo 1 Francisco Santos Leonardo 2 Jackson Maurício Lopes Costa 1 Yrla Nívea Oliveira Pereira 1 Francinaldo Soares Silva 1

\section{Flebotomíneos (Diptera, Psychodidae) de área endêmica de leishmaniose na região dos cerrados, Estado do Maranhão, Brasil}

\author{
Sandflies (Diptera, Psychodidae) from \\ an endemic leishmaniasis area in the cerrado \\ region of the State of Maranhão, Brazil
}

1 Núcleo de Patologia Tropical e Medicina Social. Departamento de Patologia da Universidade Federal do Maranhão. Praça Madre Deus 02, São Luís, MA 65025-560, Brasil. 2 Fundação Nacional de Saúde - Distrito Sanitário de Codó. Rua 1o de Maio 1879a, Centro, Codó, MA 65400-000, Brasil.
A bstract This article presents a list of ten sandfly species from the genus Lutzomyia França, 1924 found in the counties of Aldeias Altas, Capinzal do Norte, Caxias, Codó, Coel ho Neto, Timbiras, Timon and Tuntum in northeastern Maranhão, Brazil. Presence of sandflies was associated with cases of visceral and cutaneous lei shmaniasis. Some 377 specimens were captured indoors with CDC light traps and 1491 speci mens in the peri domicile. The species were: Lutzomyia cortelezii, Lutzomyia evandroi, Lutzomyia goiana, Lutzomyia intermedia, Lutzomyia lenti, Lutzomyia Iongipalpis, Lutzomyia Iongipennis, Lutzomyia squamiventris, Lutzomyia termitophila and Lutzomyia whitmani. The most abundant species was L. Iongipalpis (67.4\% and $70.2 \%$ ) followed by L. whitmani (31.0\% and $24.7 \%$ ). L. Iongipalpis was captured both indoors and outdoors in all the months studied.

Key words Psychodidae; Leishmania; Leishmaniasis; DiseaseVectors

Resumo Apresentam-se dez espécies do gênero Lutzomyia França, 1924, encontradas nos cerrados do nordeste maranhense. As capturas foram realizadas das 18h às 6h, uma vez por mês, durante nove meses, nos muni cípios de Aldeias Al tas, Capinzal do Norte, Caxias, Codó, Coel ho Neto, Timbiras, Timon e Tuntum, áreas endêmi cas das leishmanioses visceral e tegumentar. No total, foram capturados 1.868 espéci mens em armadi l has luminosas ti po CDC, sendo 377 no intradomicílio (64,7\% machos e 35,3\% fêmeas) e 1.491 no peridomi cíli o (73\% machos e $27 \%$ fêmeas). As espécies encontradas foram as que seguem: Lutzomyia cortelezii, Lutzomyia evandroi, Lutzomyia goiana, Lutzomyia intermedia, Lutzomyia Ienti, Lutzomyia Iongipal pis, Lutzomyia longipennis, Lutzomyia squamiventris, Lutzomyia termitophila e Lutzomyia whitmani. As mais abundantes no intra e no peridomicílios foram L. Iongipalpis (67,4\% e 70,2\%, respectivamente) e L. whitmani (31\% e 24,7\%, respectivamente). L. Iongipal pis ocorreu em todos os meses estudados, nas estações seca e chuvosa, e em todas as locali dades trabal hadas.

Palavras-chave Psychodidae; Leishmania; Leishmaniose; Vetores de Doenças 
Introdução

A região dos cerrados do nordeste do Maranhão tem sido, nos últimos anos, alvo das atividades de controle do Programa de Leishmaniose desenvolvido no Estado, em razão do caráter en dêmico da leishmaniose visceral (LV), e também por causa do surgimento de casos autóctones de leishmaniose tegumentar americana (LTA), especialmente nas periferias urbanas, áreas de ocupação da população de baixa renda (FNS, 1997).

O aparecimento de focos sazonais de casos de infecção em diversas áreas associa-se freqüentemente ao desmatamento desordenado para estabelecimento de atividades agropecuárias, entre outros fatores (Costa et al., 1992). Vários municípios dessa região vêm apresentando positividade para flebotomíneos e inúmeros casos de leishmanioses (FNS, 1997).

A LV no Maranhão envolve um grande contingente populacional da periferia das áreas urbanas, tanto na Ilha de São Luís, área endêmica de maior importância no Estado, como no interior, nas zonas de cerrados e de contato caatinga/cerrado. Fato inusitado, quando comparado com o número de casos nas décadas de 50 e 60 no Brasil (Nascimento et al., 1996).

O motivo para que isso ocorra talvez seja o deslocamento de grupos populacionais oriundos de outras regiões do Nordeste em busca de empregos nas grandes cidades do Maranhão, como Codó, Caxias, incluindo a capital, São Luís, cuja cobertura vegetal sofreu profundas mudanças nas duas últimas décadas. Criaramse aglomerados populacionais sem a mínima infra-estrutura sanitária, possibilitando a disseminação da doença.

Nos últimos cinco anos, vem-se estudando a fauna flebotomínica do Estado do M aranhão, em especial a da Amazônia M aranhense e a da Ilha de São Luís, onde já se determinaram mais de quarenta espécies de Lutzomyia (Rebêlo et al., 1996). O objetivo inicial é conhecer a fauna e, em uma segunda etapa, definir as espécies que transmitem agentes etiológicos das leishmanioses, nas suas diversas formas, no estado. Com este trabal ho, pretendese apresentar uma lista das espécies de Lutzomyia que ocorrem nos municípios do Distrito Sanitário de Codó - Fundação Nacional de Saúde (FNS) - MA, zona dos cerrados do nordeste do Estado.

\section{Materiais e métodos}

\section{Descrição da área}

O estudo foi realizado em al guns dos municípios que integram o nordeste do Estado do Maranhão: Aldeias Altas, Capinzal do Norte, Caxias, Codó, Coelho Neto, Timbiras, Timon e Tuntum. Esses municípios pertencem ao Distrito Sanitário da FNS com sede no município de Codó. São caracterizados por apresentarem vegetações típicas de cerradão, cerrado e zona de contato cerrado/ caatinga, resultante de uma mistura de espécies vegetais de diferentes formações. Há também forte intrusão da palmeira babaçu (Orbignya phalerata) e, em alguns pontos, da palmeira carnaúba (Copernicia prunifera). É o domínio do clima quente semi-úmido e semi-árido (IBGE, 1984) (Figura 1).

\section{Amostragem}

Os dados utilizados neste trabalho foram obtidos junto ao Setor de Entomologia do Distrito Sanitário de Codó-MA. De acordo com a rotina da equipe de entomologia da FNS, os flebotomíneos foram capturados nos meses de março, abril, junho, agosto e outubro de 1996 e fevereiro, maio, outubro e novembro de 1997, com auxílio de armadi lhas luminosas elétricas montadas no intra e peridomicílios (galinheiros e chiqueiros). Em cada mês e local, eram realizadas capturas de 12 horas ininterruptas (das $18 \mathrm{~h}$ às $6 \mathrm{~h}$ ), nos diversos municípios com problemas em relação às leishmanioses. Os espécimens capturados foram identificados, sendo posteriormente reexaminados pelos técnicos do laboratório central da FNS, em São Luís. Utilizou-se teste estatístico não paramétrico (Qui-Quadrado) para analisar as diferenças entre os flebotomíneos capturados nas estações seca e chuvosa. As diferenças foram consideradas significativas quando a probabilidade $(p)$ do erro foi inferior a $5 \%(p<0,05)$.

\section{Resultados}

No total, foram encontradas dez espécies do gênero Lutzomyia França, 1924 nos cerrados do nordeste maranhense: Lutzomyia evandroi (Lima \& Antunes, 1936); Lutzomyia goi ana Martins, Falcão \& Silva, 1962; Lutzomyia longipal pis (Lutz \& Neiva, 1912); Lutzomyia termi tophila Martins, Fal cão \& Silva, 1971; Lutzomyi a whitmani (Antunes \& Coutinho, 1939); Lutzomyia cortelezii (Brethes, 1924); Lutzomyia longi pennis (Barreto, 1946); Lutzomyia squamiventris (Lutz 
Figura 1

Mapa do estado do Maranhão mostrando as zonas fitogeográficas e os respectivos municípios onde os flebotomíneos foram capturados.

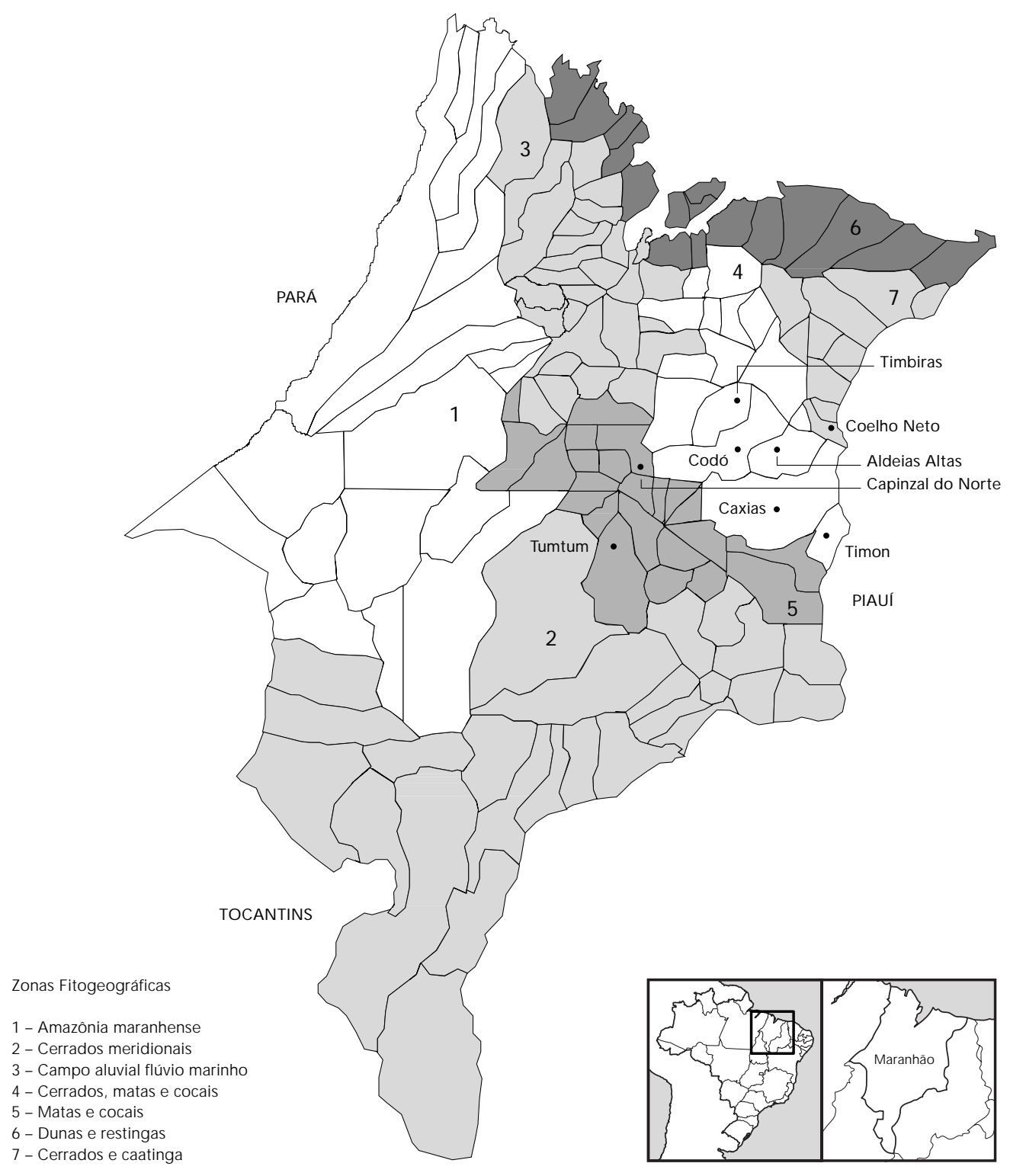


\& Neiva, 1912); Lutzomyia intermedia (Lutz \& Neiva, 1912) e Lutzomyia lenti (Mangabeira, 1938).

No peridomicílio, foram capturados 1.491 exemplares, $73 \%$ machos e $27 \%$ fêmeas. A espécie mais comum, nesse ambiente, foi L. Iongipalpis, representando cerca de 70,2\%. Seguem, na ordem de importância, L. whitmani $(24,7 \%)$ e L. Ienti (3,5\%). As demais espécies representaram juntas cerca de 1,6\% do total de indivíduos capturados (Tabela 1 ).

No intradomicílio foram encontradas apenas quatro espécies e cerca de 377 indivíduos, sendo 64,7\% machos e 35,3\% fêmeas. A espécie mais freqüente continuou sendo $L$. Iongipalpis $(67,4 \%)$, seguida por L. whitmani (31\%) (Tabela 1 ).

Os dados apresentados na Tabela 2 mostram que os flebotomíneos foram relativamente freqüentes na maioria dos meses estudados, porém as freqüências foram significativamente maiores na estação chuvosa $(p<0,05)$, que varia de janeiro a junho. O resultado reflete, sobretudo, o elevado número de espécimens L. Iongipal pis e L. whitmani capturados no mês de maio, coincidindo com o final do período chuvoso (Figura 2). L. Ienti também apresentou maior freqüência entre abril e maio. Com relação à estação seca (julho a dezembro), houve um aumento no número de espécimens amostrados nos últimos três meses. Enquanto L. evandroi, L. Ienti e L. whitmani foram encontradas durante seis meses e outras espécies ocasionalmente em um ou dois meses, L. Iongipal pis esteve presente em todos os meses e em todas as localidades estudadas (Tabela 3).

\section{Discussão}

Além de ser a espécie mais comum, tanto dentro, como fora das habitações, L. Iongipalpis também tem sido a mais encontrada nas áreas do Estado do Maranhão onde o calazar aparece de forma endêmica. Essas áreas incluem os municípios das zonas 4 e 5, de transição entre os climas semi-úmido e semi-árido, constituídas de cerrados, matas e cocais, bem como os municípios da zona 7, caracterizada pelo clima semi-árido, vegetação de cerrado e caatinga (Figura 1), com precipitação pluviométrica anual inferior a $800 \mathrm{~mm}$.

A essas três zonas fitogeográficas que caracterizam a parte nordeste do Estado pertencem os municípios do Distrito Sanitário da FNS-Codó, nos quais este trabalho foi realizado e onde o calazar alcançou níveis significativos nos últimos quatro anos (Figura 3). Só em 1994, foram registrados cerca de 245 casos; nos anos seguintes, o número de casos notificados caiu para 113 (1995), 56 (1996) e 24 (1997), segundo dados da FNS-Distrito de Codó. Existem relatos da existência nessa área de mucuras (didelfídeos) e da raposa Dusicyon vetulus, prováveis reservatórios silvestres do calazar nordestino. A partir dessas áreas mais secas, parece que o calazar vem expandindo-se para

Tabela 1

Número de espécimens de Lutzomyia coletados no intra e peridomicílios, nos municípios do Distrito Sanitário da FNS - Codó (MA), 1996-1997.

\begin{tabular}{|c|c|c|c|c|c|c|}
\hline \multirow[t]{2}{*}{ Espécies de Lutzomyia } & \multicolumn{3}{|c|}{ Intradomicílio } & \multicolumn{3}{|c|}{ Peridomicílio } \\
\hline & M & $\mathrm{F}$ & Total & $M$ & $\mathrm{~F}$ & Total \\
\hline L. cortelezii & - & - & - & - & 1 & 1 \\
\hline L. evandroi & - & 3 & 3 & 8 & 1 & 9 \\
\hline L. goiana & - & - & - & - & 1 & 1 \\
\hline L. intermedia & - & - & - & 1 & - & 1 \\
\hline L. lenti & 2 & 1 & 03 & 35 & 17 & 52 \\
\hline L. longipalpis & 164 & 90 & 254 & 736 & 310 & 1.046 \\
\hline L. longipennis & - & - & - & 4 & - & 4 \\
\hline L. squamiventris & - & - & - & - & 1 & 1 \\
\hline L. termitophila & - & - & - & 4 & 3 & 7 \\
\hline L. whitmani & 78 & 39 & 117 & 301 & 68 & 369 \\
\hline Total & 244 & 133 & 377 & 1.084 & 402 & 1.491 \\
\hline
\end{tabular}

$M=$ machos; $F=$ fêmeas. 
Número de espécimens de Lutzomyia coletados no intra e peridomicílios, nos municípios do Distrito Sanitário da FNS - Codó (MA), nas estações seca e chuvosa, 1996-1997.

\begin{tabular}{|c|c|c|c|c|c|c|c|c|c|c|}
\hline \multirow[t]{2}{*}{ Lutzomyia } & \multicolumn{5}{|c|}{ Estação chuvosa } & \multicolumn{4}{|c|}{ Estação seca } & \multirow[t]{2}{*}{ Tota } \\
\hline & Fev & Mar & $\mathrm{Abr}$ & Mai & J un & Ago & Set & Out & Nov & \\
\hline L. cortelezii & - & - & - & 1 & - & - & - & - & - & 1 \\
\hline L. evandroi & 1 & - & 1 & 3 & - & - & 1 & 3 & 3 & 12 \\
\hline L. goiana & - & - & 1 & - & - & - & - & - & - & 1 \\
\hline L. intermedia & - & - & - & - & - & - & - & - & 1 & 1 \\
\hline L. lenti & 2 & - & 10 & 39 & 1 & - & 2 & 1 & - & 55 \\
\hline L. longipalpis & 2 & 76 & 47 & 747 & 9 & 17 & 120 & 24 & 258 & 1.300 \\
\hline L. longipennis & - & - & 1 & - & 3 & - & - & - & - & 4 \\
\hline L. squamiventris & - & - & - & 1 & - & - & - & - & - & 1 \\
\hline L. termitophila & 1 & - & 4 & - & - & - & - & - & 2 & 7 \\
\hline L. whitmani & - & - & 109 & 356 & 11 & 3 & 1 & - & 6 & 486 \\
\hline Total & 06 & 76 & 173 & 1.147 & 24 & 20 & 124 & 152 & 270 & 1.491 \\
\hline Percentual & 76,3 & & & & 23,7 & & & & & \\
\hline
\end{tabular}

$p<0,05$

Figura 2

Variação sazonal da pluviosidade e dos flebotomíneos amostrados no Distrito Sanitário da FNS - Codó (MA), 1996-1997.

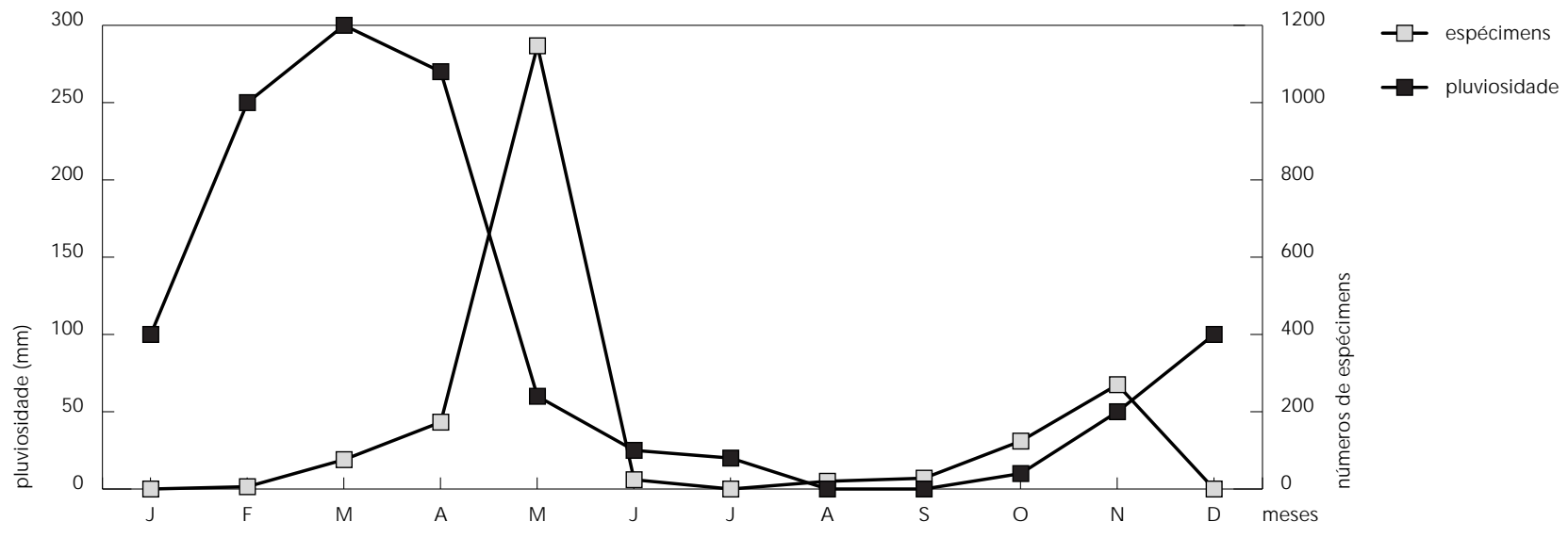

outras regiões do Estado, antigamente livres da doença.

L. Iongi pal pis é, freqüentemente, considerado um flebotomíneo de ocorrência comum em lugares pouco úmidos e um tanto montanhosos, com abundância de rochas superficiais (Forattini, 1973; Costa et al., 1990), sendo mais raro em regiões florestais e em áreas úmidas. Por esses motivos, o calazar foi, durante muito tempo, próprio de certas áreas nordestinas, de características climáticas secas ou moderadamente úmidas. Contudo, ultimamente, fatores climáticos e o tipo de vegetação parecem não funcionar mais como barreira para a expansão do calazar, haja vista a problemática da doença na Ilha de São Luís - MA (Silva et al., 1997) e na Amazônia Paraense, onde a presença desse díptero parece restringir-se às áreas 
Distribuição das espécies de Lutzomyia de acordo com os municípios trabalhados no Distrito Sanitário de Codó - MA (1996-1997).

\begin{tabular}{lcccccccc}
\hline Lutzomyia & $\begin{array}{c}\text { Aldeias } \\
\text { Altas }\end{array}$ & $\begin{array}{l}\text { Capinzal } \\
\text { do Norte }\end{array}$ & Caxias & Codó & $\begin{array}{l}\text { Coelho } \\
\text { Neto }\end{array}$ & Timbiras & Timon & Tuntum \\
\hline L. cortelezii & - & - & - & - & - & - & $\mathrm{x}$ & - \\
L. evandroi & $\mathrm{x}$ & $\mathrm{x}$ & - & $\mathrm{x}$ & - & $\mathrm{x}$ & $\mathrm{x}$ & - \\
L. goiana & - & - & - & - & - & - & $\mathrm{x}$ & - \\
L. intermedia & - & $\mathrm{x}$ & - & - & - & - & - & - \\
L. lenti & $\mathrm{x}$ & - & $\mathrm{x}$ & - & - & $\mathrm{x}$ & $\mathrm{x}$ & $\mathrm{x}$ \\
L. longipalpis & $\mathrm{x}$ & $\mathrm{x}$ & $\mathrm{x}$ & $\mathrm{x}$ & $\mathrm{x}$ & $\mathrm{x}$ & $\mathrm{x}$ & $\mathrm{x}$ \\
L. longipennis & - & - & - & - & $\mathrm{x}$ & - & - & $\mathrm{x}$ \\
L. squamiventris & - & - & - & - & - & - & $\mathrm{x}$ & - \\
L. termitophila & $\mathrm{x}$ & $\mathrm{x}$ & - & - & $\mathrm{x}$ & - & - & - \\
L. whitmani & - & $\mathrm{x}$ & $\mathrm{x}$ & $\mathrm{x}$ & $\mathrm{x}$ & - & $\mathrm{x}$ & $\mathrm{x}$ \\
\hline
\end{tabular}

Figura 3

Números de casos de leishmaniose visceral (LV) e tegumentar americana (LTA) notificados nos municípios do Distrito Sanitário da FNS - Codó (MA), 1994-1997.

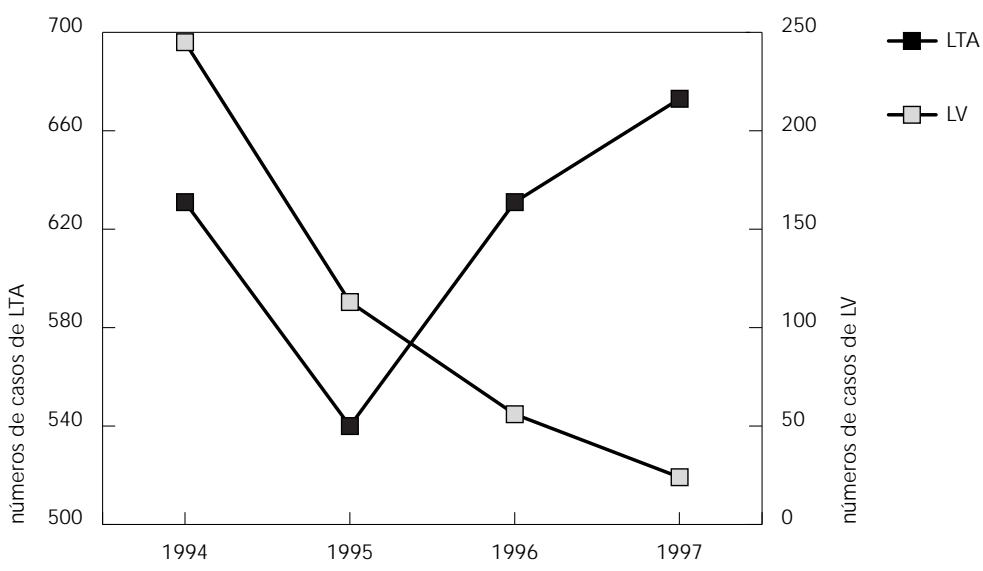

mais altas, que não estão sujeitas a inundações (Lainson, 1985; Courtenay et al., 1994).

$\mathrm{Na}$ Ilha de Marajó (PA), o ciclo de transmissão da L. chagasi é essencialmente silvestre, envolvendo a raposa Cerdocyon thous e L. Iongipal pis (Lainson et al., 1987), sendo esporádicos ou raros os casos de calazar humano, ou envolvendo o cão doméstico (Lainson, 1985). Entretanto, a llha de São Luís tornou-se um foco de calazar canino e humano, em virtude de, sobretudo, fatores históricos, apesar de possuir feições semelhantes às da Amazônia. Apresen- ta um clima semi-úmido de transição e uma vegetação que se constitui em um misto de floresta latifoliada com matas ciliares, buritizais (Mauritia vinifera) e juçarais (Euterpe oleraceae), com intrusões de babaçual (Orbygnia phal erata), manguezal e cerrados (Rebêlo et al., 1997). Entretanto, o êxodo rural interno e as migrações de outros estados nordestinos contribuíram para modificar a ecologia da l lha e desestabilizar os ecótopos da L. Iongi pal pis. Atualmente é área de alta endemicidade de calazar (Costa et al., 1995; Nascimento et al., 1996).

$M$ ais recentemente, em Imperatriz, extremo oeste da Amazônia Maranhense, têm-se notificado focos de calazar (FNS, 1997) e a existência, com freqüência elevada, de L. Iongipalpis, especialmente no peridomicílio, nos meses chuvosos. Aquela região étalvez a área de ocorrência da raposa Cerdocyon thous, comum à fauna amazônica, e que vem sendo incriminada como o hospedeiro natural da Leishmania chagasi (Lainson, 1985). Até a década de 80, não existia referência dessa enfermidade naquela região.

Nos municípios que compõem o Distrito Sanitário de Codó, foram encontradas cinco espécies de flebotomíneos (L. cortelezii, L. Iongi pennis, L. squamiventris, L. intermedia e L. lenti) que ainda não haviam sido citadas no Estado do Maranhão ou naquelas áreas onde os flebótomos são mais conhecidos com relação à estrutura faunística: Buriticupu (Amazônia Maranhense) e na Ilha de São Luís. Essas espécies têm sido encontradas apenas nas áreas mais secas do médio e baixo Parnaíba, limítrofes ao Estado do Piauí, sendo comuns à fauna nordestina e aos cerrados que avançam em dire- 
ção ao sudeste e sul do Brasil (L. squamiventris ocorre também na Bacia Amazônica).

Entre os flebotomíneos que ocorrem nas regiões dos cerrados e que estão associados com a LTA, destaca-se L. whitmani, a segunda espécie mais comum nas áreas do presente trabalho e, possivelmente, um dos principais vetores de agentes etiológi cos da LTA na região de estudo. No Estado do Maranhão já foram isoladas de pacientes as Leishmania braziliensis e L. shawi na mesma área de ocorrência de L. whitmani (Figueredo et al., 1997). Até alguns anos atrás, essa endemia restringia-se à região amazônica no Estado do Maranhão. Ultimamente, vem ampliando a sua área de ocorrência, acometendo setores antigamente livres da doença e que agora constituem-se como áreas de superposição com a LV. Nos municípios que compõem o Distrito Sanitário da FNS - Codó, a situação da LTA, nos últimos quatro anos, foi a seguinte: 631 casos (1994), 540 (1995), 631 (1996) e 673 (1997), conforme a Figura 3.

Embora não tenha sido encontrada nas áreas de LTA deste estudo, deve-se chamar a atenção para L. flaviscutellata. Esse flebotomíneo é reconhecidamente o principal vetor da Leishmania amazonensis (Lainson \& Shaw, 1974), forma responsável pelos casos humanos de leishmaniose cutânea difusa (LCD) provenientes de diversas áreas geográficas do Maranhão, inclusive a deste trabalho (Saldanha et al., 1992). Atualmente tem-se conhecimento de casos de LCD no oeste do Estado, de áreas tipicamente amazônicas; nos cerrados que ocupam toda a parte leste; nas caatingas do baixo Parnaíba e na região da baixada (Saldanha et al., 1992). São áreas ecologicamente distintas, entretanto os estudos entomológicos realiza-

\section{Agradecimentos}

À Fundação Nacional de Saúde, pelo fornecimento dos dados aqui analisados. dos em algumas delas não têm detectado a presença de L. flaviscutellata. Trata-se de fato inesperado, pois, na Ilha de São Luís, tem sido encontrado, utilizando-se o mesmo método de captura (armadilha tipo CDC). Rebêlo et al. (1998) já haviam alertado para a grande flexibilidade comportamental de L. flaviscutellata, na Il ha de São Luís. Apesar da pouca antropofilia, esse flebótomo pode realizar a hematofagia tanto dentro, quanto nos arredores das casas, bem como nas áreas silvestres.

Trata-se de um vetor importante e, como a L. whitmani, também transmite ao homem mais de um tipo de Leishmania em diferentes regiões biogeográficas (Lainson, 1988; Rangel et al., 1996). Na Amazônia Brasileira, transmite a Leishmania amazonensis e, na Venezuela, a Leishmania pifanoi. A ausência dessa espécie nas proximidades dos domicílios sugere, em trabalhos futuros, o emprego de novos métodos de captura para se detectar a presença desse díptero, em áreas de LCD no Estado do Maranhão.

Por outro lado, a presença de espécies de flebotomíneos próprias das regiões mais xerofíticas e que não têm sido encontradas nas áreas mais úmidas, como na Amazônia Maranhense, Ilha de São Luís ou na zona da Baixada Ocidental, demonstra a importância do Estado do Maranhão como área de transição entre as duas macrorregiões que caracterizam o Brasil. Pelo lado oeste, a floresta amazônica úmida, e, pelo lado leste, as savanas do nordeste seco. Sob o ponto de vista epidemiológico, o Maranhão apresenta, do lado amazônico, o predomínio das leishmanioses cutâneas, e, do nordestino, o calazar, com áreas de superposição para as duas enfermidades.
Referências

COSTA, C. H. N. \& PEREIRA, H. F., 1990. Epidemia de leishmaniose visceral no Estado do Piauí, Brasil. Revista de Saúde Pública, 24:361-372.

COSTA, J. M. L.; SALDANHA, A. C. R.; MELLO, E.; SI LVA, A. C.; SERRA-NETO, A.; GALVÃO, C. E. S.; PEDROSO, E.; SILVA, C. M. \& SILVA, A. R., 1992. Estado atual da leishmaniose cutânea difusa (LCD) no Maranhão. Revista da Sociedade Brasileira de Medicina Tropical, 25:115-123. 
COSTA, J. M. L.; VIANA, G. M. C.; SALDANHA, A. C. R.; NASCIMENTO, M. A. S.; ALVIM , A. C.; BURATTINI, M. N. \& SILVA, A. R., 1995. Leishmaniose visceral no Estado do Maranhão, Brasil. A evolução de uma epidemia. Cadernos de Saúde Pública, 11: 321-324.

COURTENAY, O.; MaCDONALD, D. W.; LAINSON, R.; SHAW, J. J. \& DYE, C., 1994. Epidemiology of canine leishmaniasis: A comparative serological study of dogs and foxes in Amazon Brazil. Parasitology, 109:273-279.

FIGUEREDO, F. V.; CUNHA, A. K.; GAMA, M. E. A. \& COSTA, J. M. L, 1997. Leishmaniose tegumentar americana (LTA), em área endêmica do Estado do Maranhão. In: XXXIII Congresso da Sociedade Brasileira de Medicina Tropical, Anais, p. 43. Belo Horizonte: Sociedade Brasileira de Medicina Tropical.

FORATTINI, O. P., 1973. Entomologia Médica. São Paulo: Editora Edgard Blücher/Edusp.

FNS (Fundação Nacional de Saúde), 1997. Relatório Anual. São Luís: Serviço de Epidemiologia/Coordenação Regional do Maranhão/ Fundação Nacional de Saúde.

IBGE (Fundação Instituto Brasileiro de Geografia e Estatística), 1984. Atlas do Maranhão. Rio de Janeiro: Superintendência de Estudos Geográficos e Sócio-Econômicos/IBGE.

LAINSON, R., 1985. Our present knowledge of the ecology and control of leishmaniasis in the Amazon Region of Brazil. Revista da Sociedade Brasileira de Medicina Tropical, 18:47-56.

LAINSON, R.; SHAW, J. J.; SILVEIRA, F. T. \& BRAGA, R. R., 1987. American visceral leishmaniasis: On the origin of Leishmania (Leishmania) chagasi . Transactions of the Royal Society of Tropical Medicine and Hygiene, 81:517.
NASCIMENTO, M. D. S. B.; COSTA, J. M. L.; FIORI, B. I. P. \& VIANA, G. M. C., 1996. Aspectos epidemiológicos determinantes na manutenção da leishmaniose visceral no Estado do Maranhão, Brasil. Revista da Sociedade Brasileira de Medicina Tropical, 29:233-240.

RANGEL, E. F.; LAINSON, R.; SOUZA, A. A.; READY, P. \& AZEVEDO, C. R., 1996. Variation between geographical populations of Lutzomyia (Nyssomyia) whitmani (Antunes \& Coutinho, 1939) sensu lato (Diptera: Psychodidae: Phlebotominae) in Brazil. Memórias do Instituto Oswaldo Cruz, 91:43-50.

REBÊLO, J. M. M.; MENDES, W. A.; COSTA, J. M. L. \& CAVALEIRO, N., 1997. Lista preliminar das espécies do gênero Lutzomyia França, 1924 (Psychodidae, Phlebotominae) do Estado do Maranhão, Brasil. Cadernos de Saúde Pública, 12:545-549.

REBÊLO, J. M. M.; ARAÚJO, J. C.; CARVALHO, M. L.; BARROS, V. L. L.; SILVA, F. S. \& OLIVEIRA, S. T., 1998. Flebótomos (Lutzomyia, Phlebotominae) da IIha de São Luís, Zona do Golfão Maranhense, Brasil. Relatório técnico-científico apresentado ao CNPq. São Luís: Universidade Federal do Maranhão. (mimeo)

SALDANHA, A. C. R.; SILVA, A. C. M.; GALVÃO, C. E. S.; SILVA, C. M. P. \& COSTA, J. M. L., 1992. Procedência de pacientes portadores de leishmaniose cutânea difusa (LCD) no Estado do Maranhão - Brasil. Revista da Sociedade Brasileira de Medicina Tropical, 25:271-273.

SILVA, A. R.; VIANA, G. M. C.; VARONIL, C.; PIRES, B.; NASCIMENTO, M. D. S. D. \& COSTA, J. M. L., 1997. Leishmaniose visceral (calazar) na Il ha de São Luís, Maranhão, Brasil: Evolução e perspectivas. Revista da Sociedade Brasileira de Medicina Tropical, 30:359-368. 